\title{
CUTANEOUS LEISHMANIASIS IN A BANGLADESHI ADULT: A CASE REPORT
}

\author{
ABDUR RAHIM ${ }^{1}$, MD. MONIRUZZAN ${ }^{1}$, RASHEDUL HASSAN ${ }^{1}$, MONIRA SARMIN $^{1}$, MD. ABDULLAH YUSUF $^{2}$, \\ BE-NAZIR AHMED ${ }^{3}$, MD. MUJIBUR RAHMAN ${ }^{1}$
}

\begin{abstract}
Cutaneous leishmaniasis is rare in Bangladesh although very few case reports are seen since last few years. But Visceral Leishmaniasis (kala azar) and PKDL are common in this region. In country like ours where tuberculosis and leprosy are more prevalent Cutaneous Leishmaniasis is very likely to be mistreated as Cutaneous tuberculosis especially lupus vulgaris or leprosy. Cases of Cutaneous Leishmaniasis (CL) are usually imported to Bangladesh from other endemic countries. A patient from an endemic area of Cutaneous Leishmaniasis, a non-healing nodulo-ulcerative lesion on exposed part of the body, dermal infiltration with lymphocytes, histiocytes and plasma cells and demonstration of intracellular parasites in lesional skin establish the diagnosis of Cutaneous Leishmaniasis. We present a case of Cutaneous Leishmaniasis in a Bangladeshi adult working in Saudi Arabia for more than 15 years. He presented with multiple ulcerative lesions on nasal bridge, right ear lobule and dorsum of right ring finger. The patient's clinical history, morphology of the lesions and laboratory analysis were consistent with Cutaneous Leishmaniasis, a rare entity for Bangladesh.
\end{abstract}

Keywords: Leishmaniasis, sodium stibogluconate, LD bodies.

Received: 05 June 2013

Accepted: 19 June 2014

\section{Introduction}

Leishmaniasis is a group of diseases caused by several species of the genus Leishmania. It has been estimated that 1.5 million new cases of Cutaneous Leishmaniasis occur annually and more than $80.0 \%$ of the total cases affect individuals in developing countries. Brazil, Iran, Afghanistan and Sudan suffer the highest prevalence and the disease is a priority for public health in all hyper endemic regions of the world $^{1}$. Human Leishmaniasis is usually classified as Cutaneous and visceral or old world type and new world type ${ }^{1}$. The species involved in old world type are L. major, L. tropica, L. aethiopica and L. donovani infantum. The species responsible for new world type of Cutaneous Leishmaniasis are L. Mexicana, $L$ brasiliensis $^{1}$. It is transmitted by the bite of female sand fly of the genera Phlebotomus in Old world, Lutzomyia and Psychodopygus in the new world. Almost all of the Cutaneous Leishmaniasis passes through common stages of papule and nodule formation, central crusting, ulceration and healing with scar formation $^{1}$. In many instances a simple test like demonstration of Amastigotes in Giemsa-stained smears from infected skin by direct microscopy can easily confirm the diagnosis. Both systemic as well as local treatments are helpful. In Bangladesh although kala azar is common, few cases of Cutaneous Leishmaniasis was documented ${ }^{2}{ }^{3}$. Here we report a case of Cutaneous Leishmaniasis, who was successfully treated with intramuscular injection of sodium stibogluconate.

\section{Case report}

A 37-year-old normotensive, non diabetic Muslim male had been working in Saudi Arabia for more than 15 years returned to Bangladesh with the complaint of multiple ulcerated skin lesion for last three months. He at first noticed some nodules which subsequently became ulcerated. He gave no history of fever, cough or any other symptoms.

On examination the ulcers were seen on nasal bridge, right ear lobule and dorsum of right ring finger. Ulcers were mildly tender, reddish, deep, crusted, $3-4 \mathrm{~cm}$ in diameter with no discharge. His vital parameters were normal, no nerve thickening, lymphadenopathy or organomegaly were found. Other systemic examinations were unremarkable.

Investigations showed, Hb-16.6 gm/dl, ESR-25 mm in $1^{\text {st }}$ hour, TC of WBC-11900/cumm, neutrophil-74\%,

1. Department of Medicine, Shaheed Suhrawardy Medical College Hospital

2. Department of Microbiology, Shaheed Suhrawardy Medical College

3. Department of Disease Control, CDC, DGHS

Address of Correspondence: Dr. Abdur Rahim, Department of Medicine, Shaheed Suhrawardy Medical College Hospital, Dhaka

Bangladesh J Medicine 2014; 25 : 78-80 
lymphocyte- $21 \%$, RBS- 80 mg/dl, S.Creatinine- $0.8 \mathrm{mg} /$ dl, SGPT-25 IU/L, ECG, CXR, Urine RME were within normal limit. Anti HIV antibody was negative. Biopsy from the skin lesion confirmed the diagnosis of Cutaneous Leishmaniasis evidenced by the presence of intra and extra cellular LD bodies.

We treated the patient with sodium stibogluconate $1200 \mathrm{mg}$ intramuscular injection for 28 days without having any complications. After 2 weeks of treatment the ulcers gradually started to heal. We did slit skin smear on day 22 and day 29 and no LD body was seen.

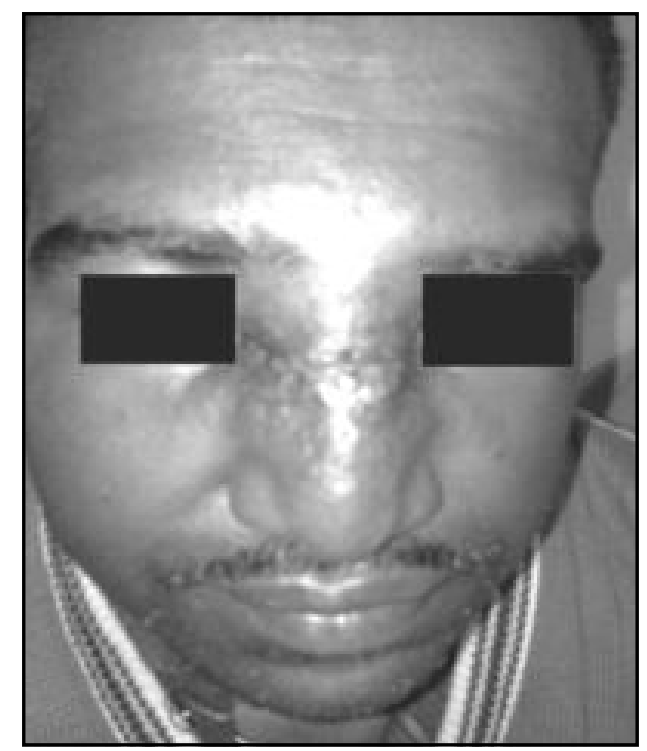

Fig.-1

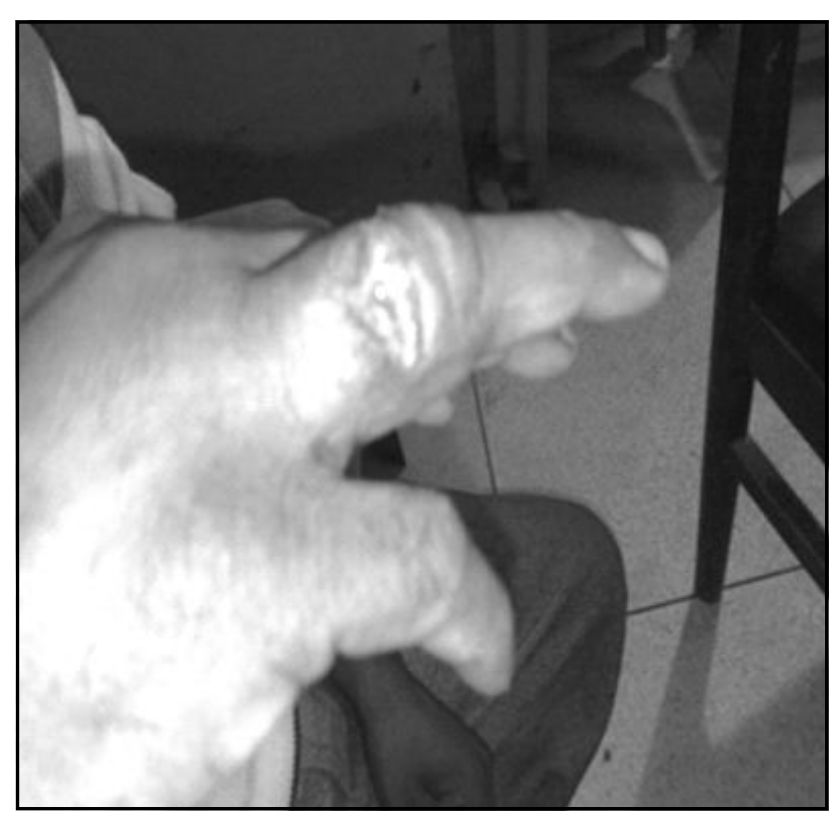

Fig.-2

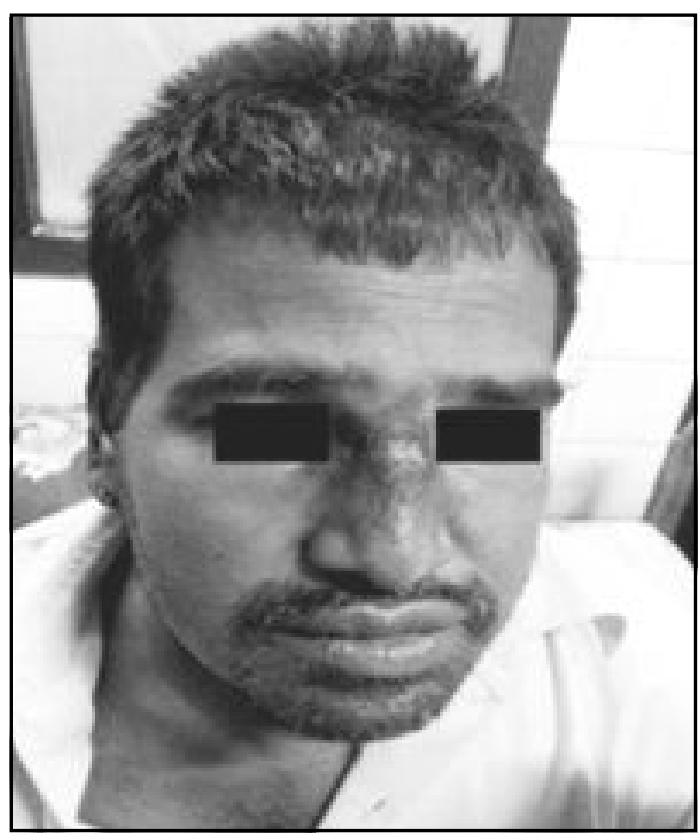

Fig.-3

\section{Discussion}

Leishmaniasis is a major world health problem. The disease is endemic in 88 countries. Brazil, Iran, Afghanistan and Sudan suffer the highest prevalence. It is caused by protozoa Leishmania. It may produce a variety of clinical syndromes varying from a simple ulcer to fatal systemic disease. Infection is transmitted by the bite of the sand fly, usually at night and outdoors. It is imported into non-endemic countries by immigrants and returning travelers as in our patient. Incubation period may range from few days to over a year. All previously uninfected individuals are susceptible for Cutaneous Leishmaniasis. One or more lesions occur in unclothed parts of the body as in our case. Clinical stages pass from papule, nodule, crusting, ulceration and finally healing with scarring. Our case presented with non healing ulcer which had started as papule subsequently progressed towards nodule formation, crusting and ulceration. Diagnosis of Cutaneous Leishmaniasis can be confirmed by demonstration of Amastigotes in Giemsa stained smears from infected skin by direct microscopy, intracellular Amastigotes in the dermis of H\&E (Haematoxylin and Eosin) stained sections from biopsy specimen, presence of Leishmania granulomas in the dermis in $H \& E$ specimens, growth of promastigotes in Nicolle-Novy-McNeal (NNN) culture medium from lesional specimens and demonstration of Leishmania DNA by PCR. The clinical diagnosis is not difficult with typical clinical features in the endemic region. 
However in non endemic countries like Bangladesh where Cutaneous Leishmaniasis is not common, it can easily be missed. Clinical patterns are poor indicators of species ${ }^{4}$. The species are distinguished by isoenzyme pattern and DNA analysis.

Most sores heal spontaneously, but their duration cannot be predicted in an individual case. Various therapeutic modalities have been used for treating Cutaneous Leishmaniasis. Intralesional pentavalent antimonials injection is one of the effective therapeutic modalities. Topical methods of treatment can be tried for simple sores. Alternate day or weekly intralesional treatment of Cutaneous Leishmaniasis with sodium stibogluconate has been shown to be more effective than daily treatments ${ }^{5}$. Uzun $\mathrm{S}$ et al, have shown the efficacy of intralesional sodium stibogluconate to be $97.2 \%$ with a low relapse rate of $3.9 \%$ and no serious adverse side effects ${ }^{6}$. However some studies have shown combination of cryotherapy with intralesional injections of sodium stibogluconate, much more effective than the use of intralesional sodium stibogluconate alone ${ }^{7}$. Systemic treatment with pentavalent antimonials is indicated for problematic sores like involvement of mucosa or cartilage, sores on the lower leg or over a joint, sores where scarring would be disabling or disfiguring and sores that might be due to parasites of the L.brasiliensis group. L. aethiopica is only sensitive to Pentamidine. Hence species characterization is also an important aspect of management. Antimonials are still the first line drug in the treatment of Cutaneous Leishmaniasis. Sodium stibogluconate (Pentostam) and meglumine antimonite (Glucantime) are essentially similar drugs which contain pentavalent antimony (Sb). Sodium stibogluconate can be administered intravenously or intramuscularly, while meglumine antimonate should only be given via the intramuscular route. The recommended dose is $20 \mathrm{mg} / \mathrm{kg} /$ day for $15-20$ days $^{8}$. Treatment with antimonials is associated with some side effects such as myalgia, as well as possible liver or cardiovascular toxicity, which fortunately is rare.

A recent study using intralesional sodium stibogluconate showed that alternate day or weekly administration of intralesional sodium stibogluconate was effective in the treatment of Cutaneous
Leishmaniasis ${ }^{5}$. Nevertheless, in our case, we were not able to identify the species due to lack of facilities for DNA analysis, hence managed successfully with intramuscular injection of sodium stibogluconate.

\section{Conclusion}

Although Cutaneous Leishmaniasis is rare in Bangladesh, clinicians should be aware of the disease while treating non healing ulcers of long duration especially if the patient is an immigrant from endemic areas.

\section{References}

1. Vega-Lopez F, Hay RJ. Parasitic worms and Protozoa- Leishmaniasis. In BurnsT, Breathnach S, Cox N, Griffiths C, editors Rook's Textbook of Dermatology(7th edition); Massachusetts; Blackwell Science 2004; 32:35-46.

2. Patwary I, Rahman M, Ahmed M, Ahmed S, Choudhury MRS. A Case of Diffuse Cutaneous Leishmaniasis in a HIV Positive Patient. $J$ Bangladesh Coll Phys Surg 2011; 29: 106-108.

3. Ahmed Z, Chowdhury SA, Bhuiyan SI. Cutaneous Leishmaniasis. Mymensingh Med J. 2009 Jul; 18(2): 260-3.

4. Jafari AK, Akhyani M, Valikhan M, Ghodsi S, Barikbin B, Toosi S. Bilateral Cutaneous leishmaniasis of upper eyelids: A case report. Dermatol Online J 2006; 12: 20.

5. Tallab TM, Bahamdam KA, Mirdad S et al. Cutaneous leishmaniasis: schedules for intralesional treatment with sodium stibogluconate. Int'l J Dermatol 1996; 35: 594-7.

6. Uzun T S, Durdu M, Culha G, Allahverdiyev AM, Memisoglu HR. Clinical features, epidemiology, and efficacy and safety of intralesional antimony treatment of Cutaneous leishmaniasis: recent experience in Turkey. J Parasitol 2004; 90: 853-9.

7. Asilian A, Sadeghinia A, Faghihi G, Momeni A, Amini HA. The efficacy of treatment with intralesional meglumine antimoniate alone, compared with that of cryotherapy combined with the meglumine antimoniate or intralesional sodium stibogluconate, in the treatment of cutaneousleishmaniasis. Ann Trop Med Parasitol 2003; 97: 493-8.

8. Dowlati Y. Treatment of cutaneous leishmaniasis (old World). Clinics in Dermatol 1996; 14:513-7. 